\title{
Bacteriological profile and antibiogram of surgical site infection/ post- operative wound infection
}

\author{
Budhani $D^{1}$, Kumar $S^{2}$, Sayal $P^{3}$, Singh $S^{4}$ \\ ${ }^{1}$ Dr Diksha Budhani, Demonstrator, Department of Microbiology, ${ }^{2}$ Dr Surinder Kumar, Head of Department, Department \\ of Microbiology, ${ }^{3}$ Dr. Pallavi Sayal, Demonstrator, Department of Microbiology, ${ }^{4}$ Dr. Sanjeet Singh, Department of \\ Community Medicine, all authors are affiliated with BPS, GMC (W), Khanpur Kalan, Sonepat, Haryana.
}

Address for Correspondence: Dr. Surinder Kumar, Head of Department, Department of Microbiology, BPS, GMC(W), Khanpur Kalan, Sonepat, Haryana.

\begin{abstract}
Background: Surgical site infections (SSI) constitute a major public health problem worldwide and are the second most frequently reported as Nosocomial infections. Surgical site infections are responsible for increasing the treatment cost, length of hospital stay and significant morbidity and mortality. Objectives: Bacteriological profile and antibiogram of Surgical Site Infection /Post-operative wound infection and to find drug useful for empirical treatment. Material and Methods: The retrospective study was conducted for a period of one year (January 2015 to Dec 2015) in the Department of Microbiology of BPSGMC Khanpur Kalan, Sonepat, Haryana. Samples were collected using sterile cotton swabs. 1687 patients clinically diagnosed of having SSIs were processed as per standard microbiological techniques. Antimicrobial susceptibility testing was done using modified Kirby-Bauer disc diffusion method. Results: Among 1687 patients screened for SSIs, $971(57.5 \%)$ were culture positive. Staphylococcus aureus (25.5\%) was the commonest organism followed by Escherichia coli (23.5\%), Citrobacter species (17.3\%) and Pseudomonas aeruginosa (9.9\%). Antimicrobial profile of gram positive isolates revealed maximum sensitivity to Linezolid and Vancomycin, whereas among gram negative isolates Imipenem, Gentamicin, Piperacillin-Tazobactam, and Amikacin were found to be most sensitive. Conclusion: Staphylococcus aureus was the commonest pathogen isolated followed by E.coli (23.5\%). Imipenem, Piperacillin/ Tazobactum, Gentamicin and Amikacin can be used as empirical treatment for gram negative bacilli and for gram positive organism Vancomycin and Linezolid can be used as empirical treatment in patients with Surgical Site Infection
\end{abstract}

Key words: Postoperative wound infection, surgical site infection, Antibiotic susceptibility testing (AST)

\section{Introduction}

Infections that occur in the wound created by an invasive surgical procedure are generally referred to as surgical site infections (SSIs). Since skin is normally colonized by a range of microorganisms that could cause infection, defining an SSI requires evidence of clinical signs and symptoms of infection rather than microbiological evidence alone [1]. The majority of SSIs become apparent within 30 days of an operative procedure and most often between the 5th and 10th postoperative days. However, where a prosthetic implant is used, SSIs affecting the deeper tissues may

Manuscript received $14^{\text {th }}$ October 2016

Reviewed: $25^{\text {th }}$ October 2016

Author Corrected: $8^{\text {th }}$ November 2016

Accepted for Publication $22^{\text {nd }}$ November 2016 occur within one year after the operation. The CDC definition 7 [1] describes three levels of SSI:

Superficial incision infection is affecting the skin and subcutaneous tissue. These infections may be indicated by localised (Celsian) signs such as redness, pain, heat or swelling at the site of the incision or by the drainage of pus.

Deep incisional, affecting the facial and muscle layers. These infections may be indicated by the presence of pus or an abscess, fever with tenderness of the wound, or a separation of the edges of the incision exposing the deeper tissues. 
Organ or space infection, involves any part of the anatomy other than the incision that is opened during the surgical procedure, for example joint or peritoneum. These infections may be indicated by the drainage of pus or the formation of an abscess detected by histopathological or radiological examination or during re-operation. Organ infection is not included within the scope of this guideline.

In addition, there may also be microbiological evidence of wound infection from cultures obtained aseptically from wound fluid or tissue. However, since skin sites are normally colonized by a variety of organisms, positive wound cultures in the absence of clinical signs are rarely indicative of SSI [1].

According to a team led by World Health Organization researchers found developing countries had much higher infection rates than the developed world it is said "poor nation face: greater hospital infection burden" Wound infection results from microbes thriving in the surgical site because of poor preoperative preparation, wound contamination, improper antibiotic selection, or the lack of ability of an immunocompromised patient to fight against infection.

Contamination of the wound is present to some extent in all incisions. A setback in recovery such as malnutrition, cardiac failure, or decreased oxygen to the tissues will deteriorate the individual and allow the infection to take hold [2 \& 3].

Skin and soft tissue infections (SSTIs) or surgical site infections are common and range in severity from minor, self-limiting, surface infections to severe diseases requiring all the resources of modern medicine.

They are also known as "The Silent Killer: Nosocomial Infections" [4].

Surgical site infections are the second most common cause of Nosocomial infections [5 \& 6]. Surgical site infections are still a threat to patients, in spite of the newer antibiotics available today. Although properly administered antibiotics can reduce postoperative surgical site infections secondary to bacterial contamination, widespread use of prophylactic antibiotics can lead to emergence of multi drug resistant bacteria. The higher rates of surgical site infections are associated with higher morbidity, mortality and increased medical expenses [5 \& 7].
The aim of the present study is to identify bacterial etiology of surgical site infections and their antibiogram to find drug useful for empirical treatment.

\section{Materials and Methods}

The present retrospective study was conducted in the Department of Microbiology at a tertiary level teaching health care facility from Jan 2015 to Dec 2015.

A total of 1687 SSIs samples were received irrespective of age and gender.

Patients of both sex who had surgical wound pus discharge, with serous or seropurulent discharge and with signs of sepsis present concurrently (warmth, erythema, induration, tenderness, pain, raised local temperature) were included [1].

Sample collection: The pus and serous fluid samples from the wounds were collected with the help of two sterile moist swab sticks from the patients, under all aseptic conditions.

Transportation and Storage: Swab sticks were transported in $2 \mathrm{ml}$ normal saline \& BHI broth to laboratory as early as possible. In case of any delay the sample were refrigerated.

Processing of samples: Two pus swabs were collected aseptically from each patient suspected of having SSI.

One swab stick was dipped in normal saline which was used for gram staining \& was incubated for 24 hours at $37^{\circ} \mathrm{c} \&$ other swab stick which was dipped in BHI was inoculated on Blood Agar \& MacConkey Agar and were cultured for $24-48$ hours at $37^{\circ} \mathrm{c}$, followed by the identification of the isolates based on their cultural characteristics and morphology with their biochemical reactions [8 \& 9].

All the isolates were tested for antimicrobial susceptibility by Kirby Bauer disk diffusion technique on Muller Hinton Agar and results were interpreted in accordance with Clinical Laboratory Standards Institute guidelines [10].

Antibiotics used for susceptibility testing were: Amikacin, Ampicillin / Sulbactam, Ceftriaxone, Ciprofloxacin, Gentamicin, Piperacillin-Tazobactum, Imipenem, Azithromycin, Vancomycin, Linezolid, Ofloxacin, Cefoxitin. 


\section{Results}

Out of 1687 samples, 971 samples were culture positive (57.5\%) (Table 1). Among 971 positive samples (56.8\%) were males (Table 1). The age wise distribution of the gender has been shown in the (Table 2) with maximum no. of culture positive samples in age 21-30 years (33.8\%) followed by 31-40 (16.06 \%) and then followed by 11-20 (12.8\%) of age group respectively. Out of 971 culture positive samples S.aureus (25.5\%) was the most common pathogen isolated followed by Escherichia coli. (23.5\%), Citrobacter spp. (17.3\%) and Pseudomonas aeruginosa (9.9\%) respectively (Table 3). Among gram negative bacilli, E.coli was most sensitive to Imipenem (88.2\%) followed by Amikacin (75.1\%) and Piperacillin Tazobactam (74.6\%) whereas for Citrobacter spp., Imipenem (75.5\%) followed by Gentamicin (44.04\%), Ciprofloxacin (42.2\%) was the drug of choice then for Klebsiella spp., Imipenem (76.5\%) followed by Gentamicin (46.9\%), Amikacin (41.9\%) was the drug of choice. For Pseudomonas aeruginosa, Imipenem (68.04\%) followed by Piperacillin Tazobactam (59.7\%), Gentamicin (57.7\%) was the drug of choice and for Enterobacter spp., Imipenem (76.7\%) followed by Amikacin (51.7\%), Piperacillin Tazobactam (50\%) showed maximum sensitivity (Table 4). Among gram positive organism, S.aureus showed maximum antibiotic sensitivity to Linezolid (96.6\%) followed by Vancomycin (95\%), Amikacin (82.5\%) whereas CONS was sensitive to Linezolid (97.8\%) followed by Vancomycin $(96.8 \%)$, and Gentamicin $(85.6 \%)$ (Table 5$)$.

Table-1: Gender wise distribution of Culture positive Patients.

\begin{tabular}{|c|c|}
\hline Gender & Culture Result \\
\hline Male & $552 / 971(56.8 \%)$ \\
\hline Female & $419 / 971(43.1 \%)$ \\
\hline
\end{tabular}

Table-2: Age wise Distribution of Culture Positive Patients.

\begin{tabular}{|c|c|}
\hline Age in year & Culture Positive \\
\hline $0-10$ & $47(4.8)$ \\
\hline $11-20$ & $125(12.8)$ \\
\hline $21-30$ & $329(33.8)$ \\
\hline $31-40$ & $156(16.06)$ \\
\hline $41-50$ & $123(12.6)$ \\
\hline $51-60$ & $98(10.09)$ \\
\hline $61-70$ & $65(6.69)$ \\
\hline $71-80$ & $28(2.8)$ \\
\hline Total & 971 \\
\hline
\end{tabular}

Table-3: Distribution of Organisms Causing Surgical Site Infection.

\begin{tabular}{|c|c|}
\hline Organism & No. of isolates (\%) \\
\hline Staphylococcus aureus & $248(25.5)$ \\
\hline Escherichia coli & $229(23.5)$ \\
\hline Citrobacter spp. & $168(17.3)$ \\
\hline Pseudomonas aeruginosa & $97(9.9)$ \\
\hline Klebsiella spp. & $81(8.3)$ \\
\hline CONS & $60(6.1)$ \\
\hline Enterobacter spp. & $56(5.7)$ \\
\hline Acinetobacter spp. & $22(2.2)$ \\
\hline Proteus spp. & $10(1.02)$ \\
\hline Total & 971 \\
\hline
\end{tabular}


Table-4: In-Vitro Antibiotic Sensitivity in Isolated Gram Negative Bacteria.

\begin{tabular}{|c|c|c|c|c|c|}
\hline Drugs & $\begin{array}{c}\text { Escherichia coli } \\
(\boldsymbol{\%})(\mathbf{n = 2 2 9})\end{array}$ & $\begin{array}{c}\text { Citrobacter spp. } \\
(\mathbf{\%}) \\
(\mathbf{n = 1 6 8})\end{array}$ & $\begin{array}{c}\text { Klebsiella } \\
\mathbf{s p p .} \\
(\boldsymbol{\%})(\mathbf{n = 8 1})\end{array}$ & $\begin{array}{c}\text { Pseudomonas } \\
\text { aeruginosa }(\boldsymbol{\%}) \\
(\mathbf{n = 9 7})\end{array}$ & $\begin{array}{c}\text { Enterobacter } \\
\text { spp. (\%) } \\
(\mathbf{n = 5 6})\end{array}$ \\
\hline Gentamicin & $\mathbf{S}$ & $\mathbf{S}$ & $\mathbf{S}$ & $\mathbf{S}$ & $\mathbf{S}$ \\
\hline Ciprofloxacin & $61(26.6)$ & $71(42.2)$ & $26(32.09)$ & $51(52.5)$ & $27(48.2)$ \\
\hline $\begin{array}{l}\text { Piperacillin/ } \\
\text { Tazobactam }\end{array}$ & $171(74.6)$ & $54(32.1)$ & $24(29.6)$ & $58(59.7)$ & $28(50)$ \\
\hline Amikacin & $172(75.1)$ & $69(41.07) 1$ & $34(41.9)$ & $52(53.6)$ & $29(51.7)$ \\
\hline $\begin{array}{l}\text { Ampicillin/ } \\
\text { Sulbactam }\end{array}$ & $69(30.1)$ & $36(21.4)$ & $22(27.1)$ & $28(28.8)$ & $12(21.4)$ \\
\hline Impinem & $202(88.2)$ & $127(75.5)$ & $62(76.5)$ & $66(68.04)$ & $43(76.7)$ \\
\hline Ceftriaxone & $53(23.1)$ & $39(23.2)$ & $18(22.2)$ & $41(42.2)$ & $15(26.7)$ \\
\hline
\end{tabular}

Table-5: In-Vitro Antibiotic Sensitivity in Isolated Gram Positive Bacteria.

\begin{tabular}{|c|c|c|}
\hline Drugs & $\begin{array}{c}\text { Staphylococcus aureus (\%) } \\
(\mathbf{n = 1 2 0})\end{array}$ & $\begin{array}{c}\text { CONS (\%) } \\
(\mathbf{n = 1 8 8})\end{array}$ \\
\hline Szithromycin & $\mathbf{S}$ & $118(62.7)$ \\
\hline Vancomycin & $75(62.5)$ & $182(96.8)$ \\
\hline Linezolid & $114(95)$ & $184(97.8)$ \\
\hline Gentamicin & $116(96.6)$ & $161(85.6)$ \\
\hline Ofloxacin & $93(77.5)$ & $133(70.7)$ \\
\hline Cefoxitin & $98(81.6)$ & $112(59.5)$ \\
\hline Amikacin & $82(68.3)$ & $130(69.1)$ \\
\hline
\end{tabular}

\section{Discussion}

Despite the advances in surgical techniques and better understanding of the pathogenesis of wound infection, SSIs management remains a significant concern for surgeons and physicians in a health care facility. Patients with SSIs face additional exposure to microbial populations circulating in a hospital set up which is always charged with microbial pathogens.

In the present study the Culture positive SSI rate was $57.5 \%$ (Table 1) whereas various other studies from India have shown the rate of SSI to vary from $6.1 \%$ to $38.7 \%$ [11,12,13 \&14]. The main Reason behind may be due to the lack of attention towards the infection control measures, inappropriate hand hygiene practices and overcrowded hospitals.

In our study, it was observed that rate of infection was higher in male patients $(56.8 \%)$ (Table 1$)$. The results were similar to a study by Vikrant Negi et al, who reported that $(74.6 \%)$ males were more commonly affected than females (25.5\%) [15]. In contrast to our study Pooja Singh. Gangania et al reveals that $20 \%$ Females shows almost equal distribution of $19 \%$ of males.

The findings in the study revealed that maximum culture positivity of the patients were with the age group 21-30 (33.8\%) years followed by 31-40 (16.06\%) years (Table 2). Similar results was showed by Pooja Singh Gangania who concluded that maximum no of SSI was in 16-45years of age group (24\%) patient. This may be due to heavy work load, stress at this age group and less number of patients [16].

S.aureus $(25.5 \%)$ was the most common pathogen isolated followed by E.coli $(23.5 \%)$ (Table 3$)$. This 
result is consistent with reports from other studies SP Lilani, Mulu W [12 \& 17]. S. aureus infection is most likely associated with endogenous source as it is a member of the skin and nasal flora and also with contamination from environment, surgical instruments or from hands of health care workers [15].

In the present study among gram negative organism, $E$. coli was Sensitive to Imipenem $(88.2 \%)$ followed by Amikacin (75.1\%) and Piperacillin/ Tazobactam (74.6\%) respectively (Table 4). The findings are consistent with the previous study conducted by $\mathrm{M}$. saleem et al who also showed that E. coli showed high sensitivity to Imipenem [18].

In this study Citrobacter spp was highly sensitive to Imipenem $(75.5 \%)$ followed by Gentamicin (44.04\%) and for Klebsiella spp., Imipenem $(76.5 \%)$ followed by Gentamicin (46.9\%) was the drug of choice (Table 4 ).

The findings are consistent with the study conducted by Jyoti Sonawane et al who also showed that Citrobacter and Klebsiella showed high sensitivity to Imipenem [19].

We observed Pseudomonas aeruginosa was most sensitive to Imipenem $(68.04 \%)$ followed by Piperacillin Tazobactam (59.7\%) (Table 4). Similar results were shown by Jyoti Sonawane et al., 2012[19].

Imipenem, Piperacillin/ Tazobactum, Gentamicin and Amikacin were found to be more efficient antibiotics against gram negative bacilli (Table 4). Similar results were observed by M. saleem et al., 2015 who showed that Amikacin, Imipenem, Piperacillin/ Tazobactum, were found to be more efficient antibiotics against gram negative bacilli [18].

In Gram positive cocci, S.aureus isolated was sensitive to Linezolid (96.6\%) followed by Vancomycin (95\%) (Table 5). This was in consistent with the study by Prem Prakash Singh et al., 2015 who also concluded that $S$. aureus was sensitive to Vancomycin (100\%), Linezolid (100\%) [20].

Linezolid and Vancomycin were found to be more efficient antibiotics against gram positive cocci (Table $5)$. This finding was in tandem with the study conducted by Vikrant Negi et al., 2015, who also reported that Vancomycin and Linezolid found to be more efficient antibiotics against gram positive cocci [15].

\section{Conclusion}

Staphylococcus aureus was the most common pathogen isolated followed by E.coli. E. coli showed maximum sensitivity to Imipenem, Amikacin, Piperacillin/ Tazobactam. In Gram positive cocci, S. aureus showed maxium sensitivity to Linezolid and vancomycin. Imipenem, Piperacillin / Tazobactum, Gentamicin and Amikacin can be used as empirical treatment for gram negative bacilli and for gram positive organism. Vancomycin and Linezolid can be used as empirical treatment in patients with Surgical Site Infection

Despite the modern aseptic procedures followed in the hospital, SSI remains as a serious problem for patients and surgeons. Hospitals serve as a reservoir for SSIs as they harbour a variety of pathogenic microbes and multi drug resistant strains. Studying the bacteriological and antibiotic susceptibility profile of SSI paves way to select the empirical antibiotic accordingly and thereby reducing morbidity and mortality and rate of SSI.

\section{Funding: Nil, Conflict of interest: None. Permission of IRB: Yes}

\section{References}

1. CDC/NHSN. Surveillance Definitions for Specific Types of Infections. 2016 April; 1-24.

2. Owings MF, Kozak LJ. Ambulatory and inpatient procedures in the United States, 1996. Vital Health Stat 13. 1998 Nov;(139):1-119.

3. Sabiston. Text book of surgery: The biological basis of modern surgical practice Beauchamp eversmattox surgical complications, (16th Ed) 199.

4. Dryden MS. Skin and soft tissue infection: microbiology and epidemiology. Int $\mathrm{J}$ Antimicrob Agents. 2009 Jul;34 Suppl 1:S2-7. doi: 10.1016/S09248579(09)70541-2.

5. Sohil Ahmed Khan, Padma G.M .Rao, Anand Rao, Gabriel Rodrigues. Survey and evaluation of antibiotic prophylaxis usage in surgery wards of tertiary level institution before and after the implementation of clinical guidelines. Indian Journal of Surgery 2006; vol. 68 (3): 150- 156.

6. Burke JP. Infection control - a problem for patient safety. N Engl J Med. 2003 Feb 13;348(7):651-6. 
7. Sasse A, Mertens R, and Sion JP, et al. Surgical prophylaxis in Belgian hospitals Estimate of costs and potential savings. J Antimicrobial Chemotherapy 1998; 41: 267-272.

8. MacFaddin J. Biochemical Tests for Identification of Medical Bacteria. 3rd ed. Philadelphia: Lippincott Williams and Wilkins; 1976.

9. Forbes BA, Sahm DF, Weissfeld AS. Bailey and Scott's Diagnostic Microbiology,10th ed, St. Louis, Misssouri, USA: Mosby Inc.; 1998.

10. Clinical and Laboratory Standard Institute. Performance Standards for Antimicrobial Susceptibility Testing. Vol. 1, No. 1, M2 A9. Pennsylvania, USA: Clinical and Laboratory Standard Institute; 2007.

11. Malik S, Gupta A, Singh PK, Agarwal J, Singh M. Antibiogram of aerobic bacterial isolates from postoperative wound infections at a tertiary care hospital in india. Journal of Infectious Diseases Antimicrobial Agents. 2011;28:45-51.

12. Lilani SP, Jangale N, Chowdhary A, Daver GB. Surgical site infection in clean and clean-contaminated cases. Indian J Med Microbiol. 2005 Oct;23(4):249-52.

13. Khan A K A, P V M, Rashed MR, Banu G. A Study on the Usage Pattern of Antimicrobial Agents for the Prevention of Surgical Site Infections (SSIs) in a Tertiary Care Teaching Hospital. J Clin Diagn Res. 2013 Apr;7(4):671-4. doi: 10.7860/JCDR/2013/5323. 2878. Epub 2013 Feb 27.

14. Chakarborty SP, Mahapatra SK, Bal M, Roy S. Isolation and identification of [14] vancomycin resistant Staphylococcus aureus from postoperative pus sample. Al Ameen J Med Sci. 2011; 4(2):152-68.
15. Vikrant Negi, Shekhar Pal, Deepak Juyal, Munesh Kumar Sharma, Neelam Sharma. Bacteriological Profile of Surgical Site Infections and Their Antibiogram: A Study From Resource Constrained Rural Setting of Uttarakhand State, India. Journal of Clinical and Diagnostic Research. 2015 Oct, Vol-9(10):DC17-DC20. DOI: $10.7860 / J C D R / 2015 / 15342.6698$.

16. Pooja Singh.Gangania, Varsha A. Singh, Sunil Sekhar Ghimire. Bacterial Isolation and Their Antibiotic Susceptibility Pattern from Post-Operative Wound Infected Patients. Indian J Microbiol Res 2015; 2(4):231-235. DOI: 10.5958/2394-5478.2015. 00020.5.

17. Mulu W., Kibru G., Beyene G., Damtie M. Postoperative nosocomial infections and antimicrobial resistance pattern of bacteria isolates among patients admitted at Felege Hiwot Referral Hospital, Bahirdar, Ethiopia. Ethiopian Journal of Health Sciences. 2012; 22(1):7-18.

18. M. Saleem, T.V. Subha, R. Balamurugan, M. Kaviraj, R. Gopal. Bacterial Profile and Antimicrobial Susceptibility Pattern of Surgical Site Infections - A Retrospective Study. Indian Journal Of Applied October 2015,Research Volume:5, Issue: 10,ISSN - 2249-555X.

19. Jyoti Sonawane, Narayan Kamath, Rita Swaminathan, Kaushal Dosani. Bacteriological profile of Surgical Site Infections and their Antibiograms in A Tertiary Care Hospital Navi Mumbai. Bombay Hospital Journal, 2010,Vol. 52, No. 3.

20. Prem Prakash Singh, Dr. Rehana Begum, Dr. Sudhir Singh, Mukesh Kumar Singh. Identification and Antibiogram of the Microorganisms Isolated from the Post operative Surgical Site Infections among the patients admitted in the hospital TMMC \& RC, Moradabad. European journal of biomedical and pharmaceutical sciences 2015,volume 2,issue4,932-942.

\section{How to cite this article?}

Budhani D, Kumar S, Sayal P, Singh S. Bacteriological profile and antibiogram of surgical site infection/ post-operative wound infection.Int J Med Res Rev 2016;4(11):1994-1999.doi:10.17511/ijmrr. 2016.i11.17. 